\title{
ONE PARAMETER GROUPS AND THE CHARACTERIZATION OF THE SINE FUNCTION
}

\author{
LAWRENCE J. WALLEN
}

(Communicated by John B. Conway)

\begin{abstract}
A real $C^{\infty}$ function on $\mathbf{R}^{1}$, all of whose derivatives and (suitable) antiderivatives are uniformly bounded, is of the form $a \sin \left(x-x_{0}\right)$. We give an abstract version of this theorem.
\end{abstract}

The characterization of the sine function given above is due to Roe (see [1]). Precisely stated, it says that if $\left\{f_{k}\right\}_{k=-\infty}^{\infty}$ is such that $f_{k}^{\prime}=f_{k+1}$ on $\mathbf{R}^{1}$ and $\sup _{k \in \mathbf{Z}}\left\|f_{k}\right\|_{\infty}<\infty$, then $f_{0}(x)=a \sin \left(x-x_{0}\right)$. Here is an abstract version.

THEOREM. Let $S_{t}$ be a strongly continuous, one-parameter group of linear operators on the Banach space $X$ with $\sup \left\|S_{t}\right\|=M<\infty$. Let $A$ be the infinitesimal generator of $S_{t}$ with domain D. Suppose $\left\{x_{k}\right\}_{-\infty}^{\infty}$ is a doubly infinite sequence of vectors in $X$ such that each $x_{k} \in D, A x_{k}=x_{k+1}$ and $\sup _{k}\left\|x_{k}\right\|<\infty$. Then $x_{0}=z_{1}+z_{2}$ where $A z_{1}=i z_{1}$, and $A z_{2}=-i z_{2}$.

Proof. Assume $x_{0} \neq 0$ and let $Y$ be the linear space of all $\left\{y_{k}\right\}_{-\infty}^{\infty}$ satisfying the hypotheses of the Theorem (the algebraic operations are, of course, componentwise). Define $\left\|\left\{y_{k}\right\}\right\|=\sup _{k}\left\|y_{k}\right\|$. Then $Y$ is a Banach space since $Y \subseteq l^{\infty}(X)$ and $A$ is a closed operator.

Now define $B$ to be the backward shift on $Y, B\left\{y_{k}\right\}=\left\{y_{k+1}\right\}$, so that $B$ is an invertible isometry. Next note that $e^{t B}\left\{y_{k}\right\}=\left\{S_{t} y_{k}\right\}$. To see this, note that the $k$ th coordinate of the left side is $\sum_{j=0}^{\infty}\left(t^{j} y_{k+j} / j !\right)$. On the other hand, $S_{t} y_{k}=\sum_{j=0}^{n}\left(t^{j} A^{j} y_{k} / j !\right)+R_{n}$ where

$$
R_{n}=\frac{1}{(n+1) !} \int_{0}^{t}(t-u)^{n+1} S_{u} A^{n+1} y_{k} d u
$$

Since $\left\|R_{n}\right\| \rightarrow 0$, equality follows. Hence $\left\|e^{t B}\right\| \leq M$ so that $\sigma(B)$ lies on the imaginary axis. But since $B$ is an isometric isomorphism, $\sigma(B)$ lies on the unit circle. Thus $\sigma(B) \subseteq\{i,-i\}$.

Consequently, $Y=Y_{i} \oplus Y_{-i}$ where $Y_{ \pm i}$ is invariant for $B$ and $\sigma\left(B \mid Y_{ \pm i}\right)= \pm i$. But on $Y_{i}, B=i I$. To see this, write $B=i I+Q$ where $Q$ is quasinilpotent. A routine calculation shows that for complex $z$ and $\varepsilon>0,\left\|e^{z Q}\right\| \leq C e^{\varepsilon|z|}$, and, of course, $\left\|e^{t Q}\right\| \leq M, t$ real. So, for arbitrary $y \in Y_{i}, y^{*} \in Y_{i}^{*}$, the entire function $y^{*} e^{z Q} y$ is of exponential type zero and is bounded on the real axis and so is constant. Hence $Q=0$. Similarly $B \mid Y_{-i}=-i I$ and the theorem follows.

To obtain Roe's result, let $X$ be the Banach space of bounded, uniformly continuous functions on $\mathbf{R}$ with the sup norm and let $S_{t}$ be left-translation by $t$. Then

Received by the editors August 1, 1986 and, in revised form, October 10, 1986.

1980 Mathematics Subject Classification. Primary 47D10, 47D05. 
if $f \in D, A f=f^{\prime}$. If now $f, f^{\prime}$, and $f^{\prime \prime}$ are bounded and continuous, then Taylor's theorem with one or two terms shows that $f \in D$ and our theorem gives $f(x)=a e^{i x}+b e^{-i x}$.

Finally, and not unexpectedly, the Hilbert space case is even more transparent. For then, by a venerable theorem of Nagy (see [2, p. 132], iA is similar to a selfadjoint operator so we will assume this at the outset. Since each $x_{k}$ is orthogonal to the kernel of $A$, we assume $A$ is invertible. But then, if $i A=\int_{-\infty}^{\infty} \lambda d E_{\lambda}$, we get $\sup _{k} \int_{-\infty}^{\infty} \lambda^{2 k} d\left\|E_{\lambda} x_{0}\right\|^{2}<\infty$ and so $E(\cdot) x_{0}$ is supported on $\{-1,1\}$.

\section{BIBLIOGRAPHY}

1. J. Roe, A characterization of the sine function, Math. Proc. Cambridge Philos. Soc. 87 (1980), 69-73.

2. J. A. van Casteren, Generators of strongly continuous semigroups, Pitman, Boston, Mass., 1985.

Department of Mathematics, University of Hawail, Honolulu, HaWail 96822 